Despite a pronounced placebo effect in the controls, the treatment group showed a uniform trend towards improvement in pain, stiffness, effusion (knee), joint line tenderness, and overall subjective and objective assessment (Fig.): at six months improvement over controls was significant for pain $(p<0.05)$ and objective assessment $(p<0 \cdot 05)$. Seven study patients and two controls noted increased bowel frequency; intermittent diarrhoea developed in three (two study patients, one control) but did not necessitate withdrawal from the trial. No changes in biochemical values or synovial fluid were observed apart from an increase in 24 hour urinary magnesium excretion from a mean (SD) of 3.9 $(2 \cdot 1) \mathrm{mmol}(\mathrm{mEq}) / 24 \mathrm{~h}$ to $5 \cdot 7(2 \cdot 5)$ $\mathrm{mmol} / 24 \mathrm{~h}$ in those taking magnesium. The degree of improvement in those taking magnesium did not correlate with renal function, initial magnesium state, or increase in urinary magnesium output. Radiological appearance of chondrocalcinosis did not change over the six month period.

The uniform trend towards improvement in those taking magnesium suggests that further studies of magnesium supplementation are warranted, especially in view of its safety in the elderly patients included in this trial. The pronounced placebo effect we observed serves to emphasise the debilitating nature of pyrophosphate arthropathy and the supportive effect of regular, interested medical attention on patients demoralised by chronic pain and stiffness.

\section{References}

1 Cheng P T, Pritzker K P H. The effect of calcium and magnesium ions on calcium pyrophosphate crystal formation in aqueous solutions. J Rheumatol 1981; 8: 772-82.

2 Bennett R M, Lehr J R, McCarty D J. Crystal shedding and acute pseudogout: an hypothesis based on a therapeutic failure. Arthritis Rheum 1976; 19: 93-7.

3 Runeberg L, Collan Y, Jokinen E J, Lähdevirta J, Aro A. Hypomagnesaemia due to renal disease of unknown aetiology. Am J Med 1975; 59: 873-81.

\title{
Problems encountered in the routine analysis of synovial fluid for crystals
}

\author{
P. R. HEARN, D. F. GUILLAND-CUMMING, AND R. G. G. RUSSELL \\ From the Department of Human Metabolism and Clinical Biochemistry, University of Sheffield Medical School, Sheffield \\ $S 102 R X$
}

Compensated polarised light microscopy is a simple technique that allows identification of crystals by determination of the sign of their birefringence based on colour change with rotation. The technique applied to crystallography of synovial fluid samples was described by Currey and Vernon-Roberts. ${ }^{1}$ Our own diagnostic service was set up in conjunction with a research project on pyrophosphate metabolism and calcium pyrophosphate crystal deposition.

The technique itself is simple if carefully carried out, but there are various problems and pitfalls. We describe some problems that we have encountered and give some hints for those setting up their own diagnostic service.

(1) Microscope. In the absence of a purpose-built polarising light microscope, most micrscopes can be converted by the addition of polarising filters and a first order red compensating filter.

(2) Slides. Ordinary glass microscope slides are used but must be as dust-free as possible; we have noted appreciable amounts of birefringent material on apparently clean slides and coverslips. Slides should be wiped repeatedly with lens tissue. Coverslips are difficult to clean because of their fragility; we rarely use them.

(3) Samples. Synovial fluid aspirates are taken without anticoagulant, as the clots which form trap crystals and considerably help the examination. Whenever possible, samples should be examined the same day, but samples stored at $4^{\circ} \mathrm{C}$ do not appear to alter during storage for up to a week. Storage at room temperature may lead to artefactual crystal growth as reported by Dieppe. ${ }^{2}$

\section{RES U LTS}

In many samples crystals were initially too small to be identified. These were tiny irregular particles or crystals often accompanied by crystals showing a Maltese cross pattern under polarised light. Many of these particles proved to be artefactual. Examination of glove powder currently used by surgeons showed the presence of numerous particles giving the same characteristic Maltese cross appearance. This powder is pure starch, which presumably enters the sample container at the time of aspiration and sample collection. A similar Maltese cross appearance is produced by lipid droplets; these can be dissolved in xylene and similar solvents and are quite commonly seen.

Fragments of cartilage, drawn up at the time of aspiration, may also cause identification difficulties, as may clots of fibrin with cells embedded in them. Irregular, weakly birefringent particles may be hydroxyapatite conglomerates, but we are unable to identify these routinely at present.

Criteria for crystal identification. Clearly, a diagnostic service must have criteria for identification of crystals. A crystal must be: bright under polarised light, exhibit birefringence, or show the required colour change on rotation of the compensating filter. It must look like a crystal-that is, have regular sides (crystal conglomerates are 
difficult to focus on), but identification by shape alone is not sufficient.

Other crystals include recently injected corticosteroids, also cholesterol which is seen as flat plates. We have seen red/brown crystals from a haemarthrosis aspirate. These were identified as bilirubin. Occasionally crystals are seen which cannot be identified, and some of these may be the alternative crystal forms of calcium pyrophosphate, ${ }^{3}$ or of calcium phosphate.

\section{References}

1 Currey H L F, Vernon-Roberts B. Examination of synovial fluid. Clinics in Rheumatic Diseases 1976; 2: 149-77.
2 Dieppe P A, Crocker P R, Corke C F, Doyle D V, Huskisson E C, Willoughby D. Synovial fluid crystals. $Q J$ Med 1979; 48: 533-53.

3 Hearn P R, Russell R G G. Formation of calcium pyrophosphate crystals in vitro: implication for calcium pyrophosphate deposition diseases (pseudogout). Ann Rheum Dis 1980; 39: 222-7.

\title{
New methods for identification of crystalline material in joints
}

\author{
D. V. DOYLE AND P. R. CROCKER
}

From the Department of Rheumatology, St Bartholomew's Hospital, London EC1A $7 B E$

The identification of microcrystalline material in joint fluid and tissues by conventional electron microscopical (EM) methods requires about 48 hours' specimen preparation. We have developed a series of methods that, in the case of joint fluids, allows the rapid preparation of fresh specimens for EM examination and, in the case of tissue specimens, enables paraffin-embedded sections to be examined directly by scanning electron microscopy (SEM).

SYNOVIAL FLUIDS

(1) Cytocentrifugation.

Transmission electron microscopy (TEM) system. The fluid is cytocentrifuged onto coated EM grids, which are then examined by light microscopy (LM) to select the most suitable monolayer for examination by TEM. Crystalline material, where detected, is analysed by $x$-ray energy spectroscopy (XES).

Scanning electron microscopy (SEM) system. The fluid is cytocentrifuged onto a standard perspex slide $(7.6 \mathrm{~cm} / 2.5 \mathrm{~cm})$, which is then placed in the microscope for examination and analysis. The specimen on the perspex slide may also be microincinerated at low temperature to remove organic material, leaving crystals clearly exposed for examination.

Scanning electron microscopy with back-scattered electron imaging facility $(B E I)$. The specimen is prepared as for SEM, but the BEI facility enables rapid location of crystalline material by atomic number differences without the need for microincineration.

\section{(2) Millipore filtration.}

SEM system. Negative pressure filtration of synovial fluids is used to collect particulate material on the surface of a millipore filter. The filter is placed on a graphite stub in the bulk holder of the scanning electron microscope, and the surface examined by SEM and XES.

SEM system with BEI facility. The preparation is as for SEM, but the BEI facility enables rapid location of crystalline material.

\section{JOINT TISSUES}

Routine paraffin block sections are used. Standard $5 \mu \mathrm{m}$ sections are cut and mounted on perspex slides.

SEM system. The tissue section on the perspex slide is examined by light microscopy and the topography is noted. The section is then microincinerated at low temperature to expose particulate material for examination by SEM and XES.

$S E M$ system with $B E I$ facility. The preparation is as for SEM, but microincineration is unnecessary. Direct examination by BEI and XES is possible.

The identification of microcrystalline material in synovial fluid on articular tissue specimens by conventional EM methods is impractical for routine diagnostic use because it is extremely time consuming. The tissue specimen is cut into very small blocks, which must then be fixed, embedded, and sectioned. This procedure takes at least 48 hours and as the specimen blocks are very small and tissue deposits of crystals are often patchily distributed, the specimen selected for examination may well be devoid of crystalline material.

Our methods used standard laboratory fixation and preparation techniques. The specimens, apart from those involving millipore filtration, may be examined initially by light microscopy to determine whether EM examination is likely to be worthwhile. These techniques are a significant advance on traditional electron microscopical methods used for the detection of crystalline material in joint fluids and tissues. 